\title{
BMJ Open Interventions to facilitate return to work in adults with chronic non-malignant pain: a protocol for a systematic review and network meta-analysis
}

Mathilda Björk, ${ }^{1}$ Björn Gerdle, ${ }^{1}$ Gunilla Liedberg, ${ }^{1}$ Frida Svanholm, ${ }^{1}$ Marco Solmi, ${ }^{2}$ Trevor Thompson, ${ }^{3}$ Anna Chaimani, ${ }^{4}$ Elena Dragioti (iD ${ }^{1}$

To cite: Björk M, Gerdle B, Liedberg G, et al. Interventions to facilitate return to work in adults with chronic nonmalignant pain: a protocol for a systematic review and network meta-analysis. BMJ Open 2020;10:e040962. doi:10.1136/ bmjopen-2020-040962

\section{- Prepublication history and} additional material for this paper is available online. To view these files, please visit the journal online (http://dx.doi.org/10. 1136/bmjopen-2020-040962)

Received 27 May 2020 Revised 16 September 2020 Accepted 21 October 2020

\section{Check for updates}

(c) Author(s) (or their employer(s)) 2020. Re-use permitted under CC BY-NC. No commercial re-use. See rights and permissions. Published by BMJ.

${ }^{1}$ Pain and Rehabilitation Centre, and Department of Health, Medicine and Caring Sciences, Linköping University, Linkoping, Sweden

${ }^{2}$ Neurosciences Department, University of Padua, Padua, Italy

${ }^{3}$ School of Human Sciences, University of Greenwich, London, UK

${ }^{4}$ Research Center of Epidemiology and Statistics Sorbonne Paris Cité (CRESS UMR1153), INSERM, INRA, Université de Paris, Paris, France

Correspondence to

Dr Elena Dragioti;

elena.dragioti@liu.se

\section{ABSTRACT}

Introduction Work absenteeism due to chronic nonmalignant pain (CNMP) is a major societal and individual cause of concern that requires effective treatments. Objective We present a protocol for a systematic review and network meta-analysis (NMA) aiming to compare available interventions for return to work (RTW) in adults with CNMP.

Methods and analysis PubMed, Embase, PsycINFO, Web of Knowledge and Cochrane Central Register of Controlled Trials databases will be searched till 31 August 2020 for randomised controlled trials (RCTs) examining interventions for RTW outcomes among patients with CNMP. Two independent investigators will search the databases, perform data extraction and assess the methodological quality of the selected RCTs. The primary outcome will be RTW, if possible, full-time or part-time after work absence due to chronic pain from baseline to the last available follow-up. Secondary outcomes will include self-reported workability or work capacity, or self-reported physical functioning and quality of life as measured by any validated scale. Pairwise meta-analysis and NMA will be conducted for each outcome using a random-effects model. For the primary outcomes, we will also obtain the ranking of all competing interventions within each NMA using surface under the cumulative ranking curve. The assumption of coherence (ie, that direct and indirect evidence are in statistical agreement) will be examined using both a local and a global approach. We will also conduct subgroup and meta-regression analyses, whenever feasible, to investigate the unexplained variation in effect size. The comparison-adjusted funnel plot will be used to evaluate small-study effects. The overall quality of evidence will be rated with the Confidence in Network Meta-Analysis tool. Data analysis will be conducted using Stata V.16.0.

Ethics and dissemination This systematic review does not require ethical approval since it will not disseminate any private patient data. The results of this study will be disseminated through peer-reviewed publication. PROSPERO registration number CRD42020171429.

\section{INTRODUCTION}

Work absenteeism due to chronic nonmalignant pain (CNMP) is a considerable
Strengths and limitations of this study

- We will conduct a systematic review and network meta-analysis (NMA) examining the best intervention option to help people with chronic nonmalignant pain (CNMP) get back to work.

- Moderate-to-large heterogeneity, risk of publication bias and important statistical incoherence might limit the type of interventions that can be included in the analysis.

- A limitation could also be the differences in social security systems in different countries and the lack of equal outcomes and data sources.

societal and individual problem requiring effective treatments. ${ }^{1}{ }^{2}$ CNMP leads to decreases in work productivity and quality of life and represents a significant psychological impact on people and economic strain on society. ${ }^{3-6}$ It is estimated that the global prevalence of general chronic pain with at least moderate pain intensity is approximately $20 \%,^{7}$ and a quarter of pain sufferers of active working age is absent from work. ${ }^{8}$ The National Health Interview Survey found that nearly 40 million adults (17.6\% of all American adults) reported having severe pain, that is, back pain, fibromyalgia, neck pain and osteoarthritis. ${ }^{9}$ Importantly, chronic low back pain is one of the leading causes of disability. ${ }^{10}$ Adding to this, the total direct and indirect costs of chronic pain, including loss of healthrelated quality of life, are considerable. In Canada, for example, these have been estimated at $\$ 1742$ per person, with higher costs in those with more severe pain and activity limitations. ${ }^{11}$

Effective intervention options for CNMP pose a great challenge among healthcare workers. Research data show that treatment policies such as the prescription of bed rest and opioids are not optimal because such 
policies are associated with higher long-term disability. ${ }^{12} 13$ Therefore, clinical practice guidelines have focused on the importance of returning to normal activities as the most optimal strategy of recovery from chronic pain conditions. ${ }^{14}$ Emerging evidence also stresses the importance of return to work (RTW) as one of the main goals of treatment, ${ }^{15-19}$ since RTW can be regarded as an indicator of better health status and recovery. ${ }^{20}$ Hence, RTW is a core outcome, not only for society but also for chronic pain sufferers themselves. As indicated above, the loss of productivity due to CNMP is enormous, ${ }^{56}$ and thus it is essential to implement cost-effective interventions to facilitate RTW. ${ }^{15-19}$ Moreover, work presenteeism is associated with an array of physical and mental health benefits, ${ }^{21}{ }^{22}$ whereas work absenteeism is associated with an array of unfavourable physical and mental health outcomes, leading to a worse financial situation. ${ }^{23-25}$

Currently, the benefits of RTW interventions remain uncertain. A recent systematic review of the effectiveness of RTW interventions delivered in the workplace found mixed evidence of the impact of these interventions on lost time and work functioning. ${ }^{16}$ Previous systematic reviews within this topic have also mainly focused on specific interventions and on specific pain populations. ${ }^{17}{ }^{18} \mathrm{~A}$ systematic review by Schaafsma et $a l^{17}$ could not find an effect of light or intensive physical conditioning programmes on the reduction of sickness absence, compared with usual care or other exercise programmes. Furthermore, Norlund $e a^{18}$ found limited evidence linked also to publication bias from randomised controlled trials (RCTs) for the effectiveness of multidisciplinary programmes on RTW for people on work absence due to low back pain. The latter is supported by the work of Kamper et al. ${ }^{26}$ Another systematic review by Vogel $e t$ $a l^{19}$ found no benefits of RTW coordination programmes for workers on work absence for at least 4 weeks compared with usual practice. Moreover, all the available evidence is based either on pairwise meta-analyses or on systematic reviews without quantifiable synthesis of data, leading to a lack of an established hierarchy for the best intervention option. ${ }^{27-29}$ In addition, head-to-head comparisons are limited in the available studies. ${ }^{17} 1829$ Consequently, a broad evaluation of the effectiveness of RTW interventions integrating direct and indirect comparisons from $\mathrm{RCTs}^{28}$ that can provide a clinical ranking is imperative for the treatment of patients with CNMP. As such, our objective is to evaluate the comparative effectiveness of RTW interventions through a systematic review and network meta-analysis (NMA) based on all eligible RCTs. NMA is a generalisation of pairwise meta-analysis that allows the comparison of all pairs of interventions included in a network for the same condition. ${ }^{28}$ NMA extends the principles of meta-analysis to the evaluation of multiple treatments in a single analysis, thus establishing a hierarchy of the best interventions for a particular condition. ${ }^{28} 30$ This is achieved by combining the direct and indirect evidence. Direct evidence refers to the evidence obtained from RCTs; for example, in a trial comparing treatments
$\mathrm{A}$ and $\mathrm{B}$, direct evidence is the estimate of relative effects between A and B. Indirect evidence refers to the evidence obtained through one or more common comparators. In other words, NMA has the potential to make treatment estimates for an entire treatment network instead of scanning each individual pairwise comparison and to more explicitly 'rank' treatments using summary outputs. ${ }^{27} 30$ The study is called NOVEL (returN to wOrk interVentions nEtwork meta-anaLysis).

\section{METHODS}

\section{Study design and registration}

This protocol is reported following the Preferred Reporting Items for Systematic Review and Meta-Analysis (PRISMA) Protocols recommendations (online supplemental file 1$)^{31}$ and the Cochrane Comparing Multiple Interventions Methods Group recommendations for NMA protocols. ${ }^{32}$ It is also registered on PROSPERO. We will use the PRISMA extension statement for NMA to prepare the final report of this study. ${ }^{33}$

\section{Eligibility criteria}

\section{Types of participants}

Adults ( $\geq 18$ years) of both sexes who have a paid job (part-time or full-time), irrespective of the position, organisation or type of employment contract at the time of diagnosis, and who are absent from work due to CNMP will be eligible for inclusion. In this study, CNMP will be defined as an umbrella term that will include chronic/ persistent nociceptive, nociplastic and neuropathic pain (see figure 1 for definitions) conditions, for example, chronic neuropathic pain, chronic low back pain, chronic neck pain including whiplash-associated disorders, chronic widespread pain, fibromyalgia, myofascial pain syndromes, Ehlers-Danlos syndrome and hypermobility syndromes, and osteoarthritis, and the conditions lasting at least $\geq 3$ months according to the International Association for the Study of Pain (IASP) ${ }^{34}$ or a physician clinical diagnosis. Diagnostic criteria as depicted above for CNMP should be established and described in eligible studies. Participants with CNMP and mental disorder comorbidity will be also included.

Participants with acute and subacute pain (ie, <3 months pain) will be excluded. Whenever both subacute and chronic pain populations are included in the same study, we will keep the study only if at least $75 \%$ of participants have had a diagnosis of CNMP. ${ }^{35}$ Whenever the relative proportion is not reported or the trials include mixed populations, we will try to determine whether data on the eligible subset can be extracted separately. We will exclude pain conditions without a chronic/persistent nociceptive, nociplastic and neuropathic pain basis. We will also exclude adults with CNMP who do not have a paid job at the time of the study, adults with CNMP on extended work absence due to it (i.e. $>3$ months) or those likely to become unemployed (including not normally in paid employment or student) within the next 12 months, 


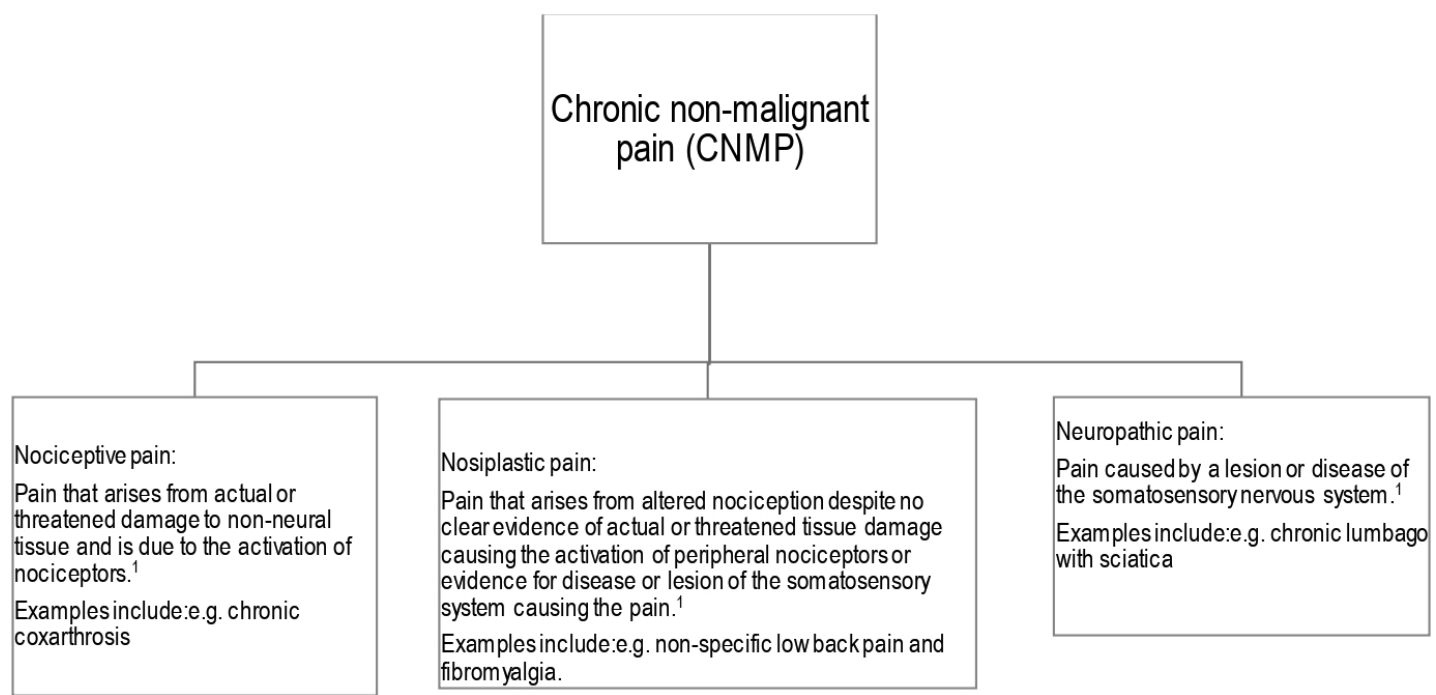

Figure 1 Definitions of nociceptive, nociplastic and neuropathic pain. Patients can have a combination of nociceptive and nociplastic pain. International Association for the Study of Pain (IASP). ${ }^{34}$ Part III: pain terms, a current list with definitions and notes on usage (pp. 209-214) In Classification of chronic pain, second edition, IASP Task force on taxonomy, edited by H. Merskey and N. Bogduk, IASP press, Seattle, 1994.

either planning to retire or take early retirement (through choice or ill health).

\section{Types of interventions and comparators}

This study will investigate comparisons of any type of intervention aiming to facilitate RTW, excluding those interventions using brief advice. We will also include interventions where RTW is not the primary outcome if at least one RTW outcome (even secondary) is reported. Since the expected interventions may focus on different RTW factors, that is, physical, psychological and social factors (eg, coping skills, workplace adjustments), ${ }^{36}$ we will group them into intervention classes to allow us to compare the relative effects of intervention classes, as previously reported in the literature ${ }^{29} 37$ :

- Psychological interventions, including cognitive behavioural therapy, problem-solving therapy and mindfulness-based stress reduction.

- Educational and self-management interventions, including pain education, self-regulatory skills such as progressive muscle relaxation and lifestyle changes.

- Work-related interventions, including vocational/ job programmes, workplace or occupational interventions.

- Physical activity/exercise interventions, including yoga, tai chi, Pilates and motor control exercise.

- Interdisciplinary pain interventions, including interventions provided by a multidisciplinary team collaborating in assessment and treatment using a shared biopsychosocial model and goals.

- Pharmacological interventions, including opioids and antidepressants, alone or in combination.

- Non-invasive modalities, including transcutaneous electrical nerve stimulation and spinal manipulative therapy.
- Surgical approaches and invasive modalities, including spinal cord stimulation, acupuncture, myofascial trigger point injections and epidural corticosteroid injections.

- Policy and healthcare system strategies/programmes, including health-focused service coordination or work modification strategies.

- Combined rehabilitation interventions as described above, including, for example, medication plus physical activity interventions.

We will exclude interventions using nutritional remedies or vitamins as monotherapies, traditional Chinese medicines and/or alternative therapies other than acupuncture because there is no or very little evidence of their effectiveness of these interventions, and investigative studies are typically of low quality. ${ }^{29} 37$

We will compare any type of intervention with another active intervention as specified above or with control (eg, placebo, no intervention, standard care (treatment-asusual) or a wait list control).

\section{Outcomes}

The primary outcome will be RTW after work absence due to CNMP. In this study, RTW will be defined as having part-time or full-time paid or supported employment without consideration of the job demands or working hours. ${ }^{38}$

RTW will be assessed as the rate of time until first RTW (as it is the most commonly used RTW outcome internationally) ${ }^{39}$ among a group after their allocation to RTW therapy or control intervention, the total duration of work absence due to CNMP over a given period, work status (working/not working) or sick-listed status (yes/ no) at a point in time, and recurrences of work absence due to CNMP by self-report or based on information 
collected from organisational or system record measurements. We will extract outcome data from baseline to the last available follow-up. In the case of multiple follow-up measurements, we will extract follow-up data close to 12 months. RTW outcomes will be plotted as either mean difference or standardised mean difference (SMD) and/or OR, depending on reported binary or continuous available measurement data. Secondary outcomes will include self-reported workability or work capacity, or self-reported physical functioning and quality of life measured by any validated scale, as described in original studies. In this study, we decided not to focus on measures of pain because a protocol of NMA is already designed and published. ${ }^{29}$ In addition, pain relief is out of scope of this NMA. We will extract secondary outcome data from baseline to the last available follow-up. In the case of multiple follow-up measurements, we will extract follow-up data close to 12 months.

\section{Type of studies}

All RCTs describing RTW outcomes of interventions for CNMP will be considered. Only RCTs with a parallelrandomised or a cluster-randomised design will be included. We will exclude quasi-randomised trials and cross-over trials if the latter is reported because the poor reporting of cross-over trials may hamper attempts to perform a meta-analysis using the available methods. ${ }^{40}$ Other study designs not pertained to RCTs, for example, cohort, case-control and registry studies, will also be excluded due to high risk of bias in such studies in terms of lacking randomisation. There will be no limitations on the number, format methods and dosage of the interventions.

\section{Search strategy}

We will search several databases from inception to 31 August 2020 for RCTs that examined interventions for RTW outcomes among patients with CNMP. We anticipate completing the study on 31 December 2020. A comprehensive literature search will be run in PubMed, Embase, PsycINFO, Web of Knowledge and Cochrane Central Register of Controlled Trials databases. The search strategy will be developed by three members of the team (ED, MB and $\mathrm{BG}$ ), with expertise in systematic reviews and clinical experience on the topic. It will comprise the combination of subject headings and key terms based on the following: RTW, chronic pain intervention, vocational rehabilitation, rehabilitation, work, work rehabilitation, job programme, chronic pain, chronic musculoskeletal pain and widespread pain. Search terms will be combined with Boolean operators "AND" and "OR" as follows: (Return to work OR Work, Return to OR Back-to-Work OR Return-to-Work OR Back to Work OR RTW OR Chronic pain Intervention OR Vocational rehabilitation OR Rehabilitation OR Work OR Work rehabilitation OR Job program) AND (Chronic pain OR Chronic Musculoskeletal Pain OR Widespread Chronic Pain). Whenever feasible, we will additionally use the Cochrane Highly
Sensitive Search Strategy for identifying randomised trials in various databases, (https://work.cochrane.org/rctfilters-different-databases). Details of search strings of the PubMed database are displayed in online supplemental file 2. We will also search for published, unpublished and ongoing trials in two clinical trial registries, namely, ClinicalTrials.gov and WHO International Clinical Trials Registry Platform. Two independent investigators (GL and ED) will search the databases. In cases of discrepancy, a third investigator (BG) will be consulted until an agreement can be reached. The references of relevant published systematic reviews and meta-analyses will also be hand-searched for additional articles missed by electronic search. There will be no language restrictions for the literature search; however, RCTs that are not possible to be translated into English will be excluded from quantitative analysis.

\section{Selection of studies}

After removing duplicates, two reviewers independently will screen the titles and abstracts. The screening will be performed using EndNote (EndNote V.X9; Thomson Reuters, San Francisco, California, USA). The full texts of potentially eligible studies will be retrieved and will be further assessed for eligibility. An overview of the eligibility screening process is shown in figure 2. RCTs fulfilling the eligibility criteria will be included, and reference lists of these trials will be also checked. If the literature is recognised as multiple submissions or duplicate publications, we will compare the differences between the two articles and retain the more detailed one. We will use the PRISMA flow diagram to outline the literature search and evaluation process of selected RCTs. Any discrepancies in selecting the trials will be resolved by discussing it with a third reviewer, if needed.

\section{Data collection process}

Data of eligible RCTs will be then extracted in a standardised Microsoft Excel form. One member of the review team will perform data extraction and coding, with $100 \%$ of all data abstraction checked for accuracy by a second member of the review team. The following data will be extracted (see table 1):

- The baseline characteristics of the eligible studies, including authors' names, year of publication, country setting, mean age, percentage of women/ men, chronic pain duration and duration of work absence due to CNMP.

- Diagnosis/pain conditions and diagnostic criteria (IASP, clinical, International Classification of Diseases (ICD)-10 or ICD-11, etc), intervention/comparator and type of outcome measurements (self-reported/ records).

- Information of interventions in detail (ie, dose, duration and delivery/format method).

- Metric measures information, including number of treatment; number of control event cases; event 
Title or Abstract meets all eligibility criteria according to PICO format?

P: Adult (18 years or older) patients with CNMP (eg. chronic/persistent nociceptive, nociplastic, andneuropathic pain basis more than three months), which are employed (full or par-time) and they are absent from work due to CNMP i.e.<three months

I: Any intervention excluding interventions using brief advise, nutritional remedies or vitamins a smonotherapy, tra ditional Chinese medicines and/or alternative therapies other than acupuncture.

C: a nother active intervention or control

O: RTW after work absence due to CNMP

Study type: RCT (parallel-randomized or a cluster-randomized design)

Yes, or unclear

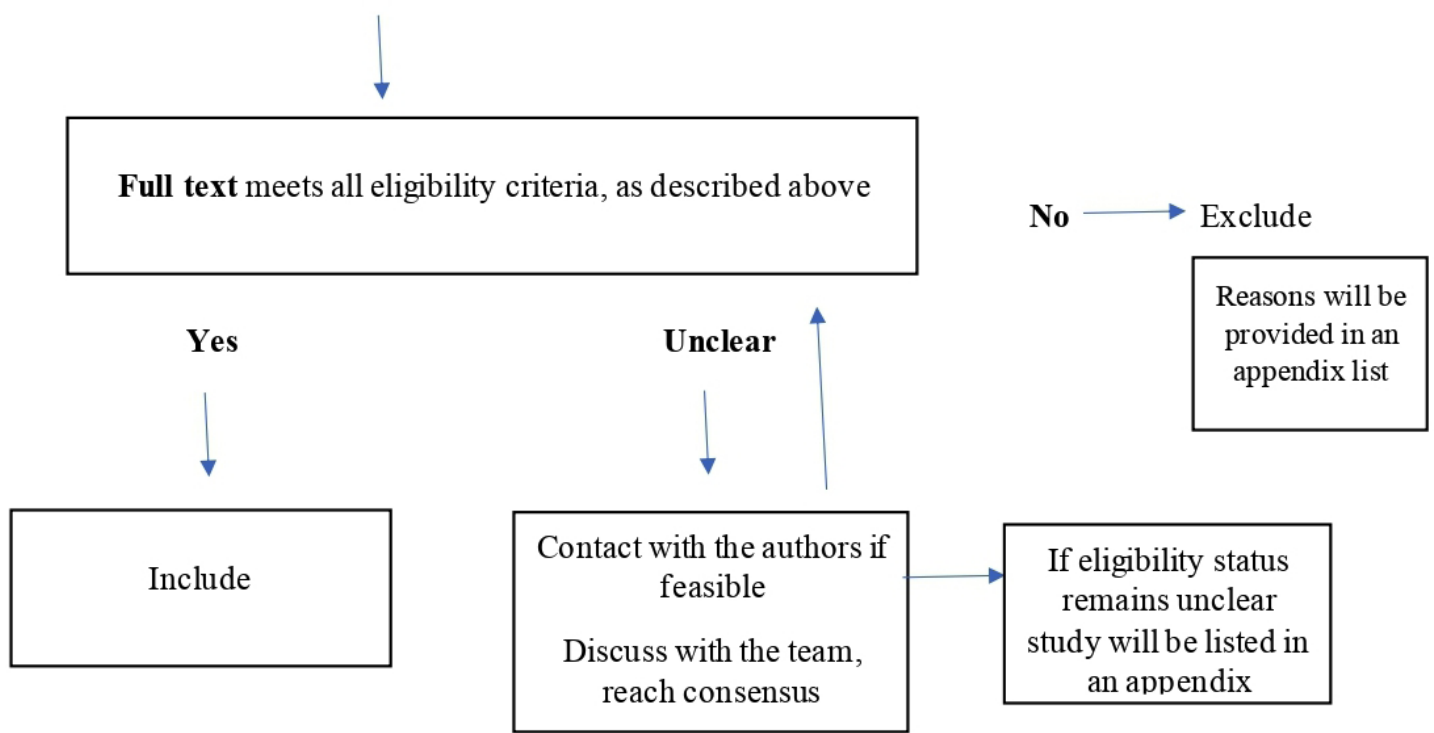

Figure 2 The eligibility screening process. CNMP, chronic non-malignant pain; PICO, patients, interventions, comparator, outcome; RCT, randomised controlled trial; RTW, return to work.

controls; mean and SD, SE or CI, or median and IQR; and sample size.

- RTW definition (the scale used to define or measure RTW), as well as the secondary outcomes from baseline to the last available follow-up.

In the case of cluster RCTs, we will extract data considering the clustering, that is, adjusted results. If adjusted results are not available, then we will extract unadjusted results and will approximate the adjusted estimates by dividing the sample size with the design effect (ie, $1+(M-1) \times \mathrm{ICC}$, with $M$ being the average cluster size and ICC the intracluster correlation coefficient), whenever feasible. ${ }^{41}$

\section{Dealing with missing data}

To obtain missing data, we plan to contact the authors of the original studies. However, after contacting the authors at least two times, studies with insufficient data will be excluded. For continuous outcomes, if SDs are missing we will calculate them from SEs, CIs or other measures. ${ }^{42} 43$
For large enough studies (eg, 50 participants per arm), we will also calculate the mean and SD for the continuous outcomes that will be reported as median and IQR using specific formulae. ${ }^{44}$

\section{Assessment of methodological quality}

We will assess the risk of bias within individual studies using the revised Cochrane risk of bias (RoB V.2.0) tool for RCTs. ${ }^{45}$ This rates the potential for study bias arising from the randomisation process, deviations from the intended intervention, missing outcome data, measurement of outcomes and selective reporting, with the overall risk of bias for each study being graded as low, high or some concerns. Two reviewers will independently assess biases per RCT.

\section{Data synthesis and statistical analysis}

We will generate descriptive statistics for trial and study population characteristics (clinical and methodological) across all eligible trials and across the available direct 


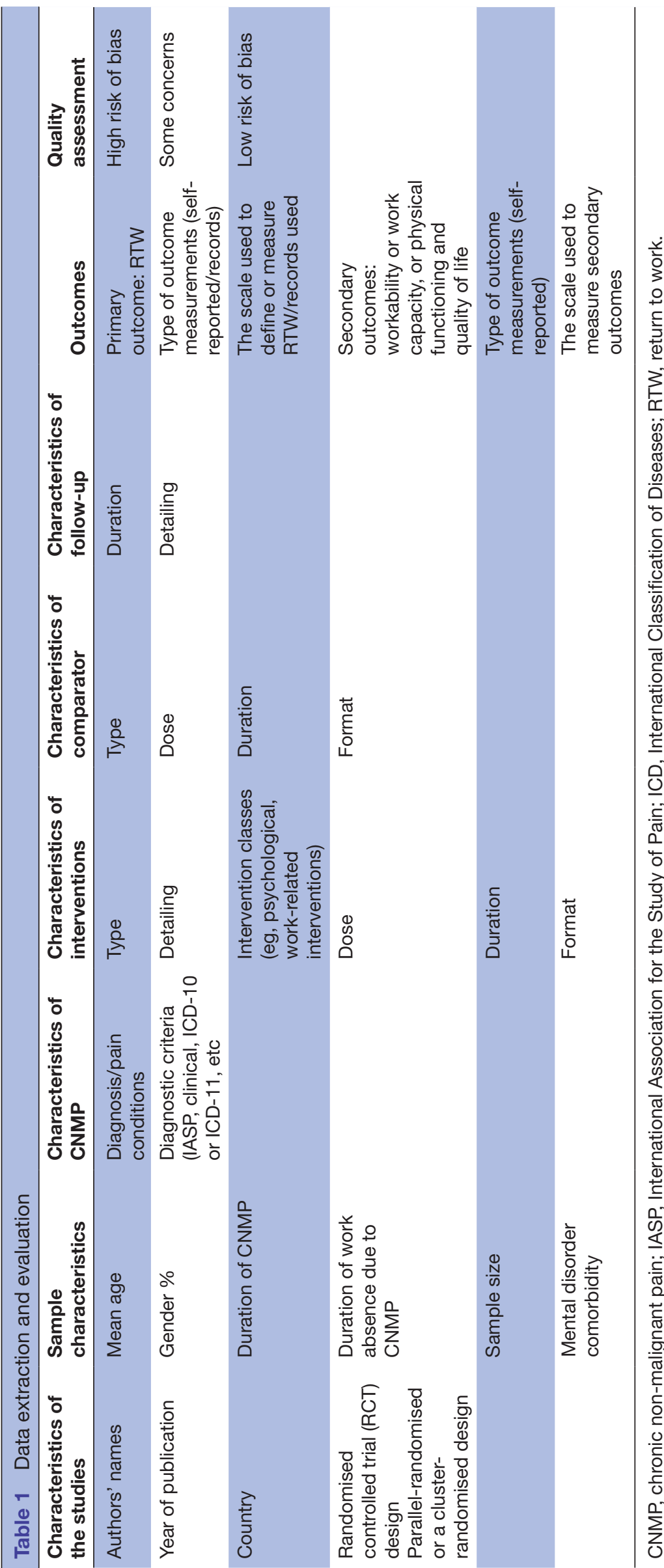

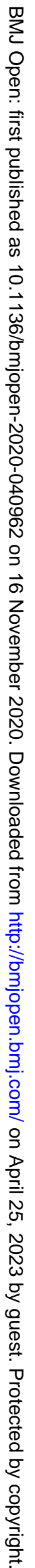


comparisons to investigate whether clinical homogeneity and transitivity, respectively, are likely to be plausible. The latter is the fundamental assumption of NMA and implies that one can validly learn about $\mathrm{B}$ versus $\mathrm{C}$ through A using studies comparing A versus B and studies comparing A versus C. ${ }^{46}$

For each pairwise comparison between the different interventions, the OR will be calculated as the effect size for dichotomous outcomes and as the SMD for continuous outcomes, both with a 95\% CI. Pooled ORs and 95\% CIs will be calculated using the inverse variance method.

\section{Pairwise meta-analysis}

We will first perform pairwise meta-analyses synthesising studies that compare the same pair of interventions for the same chronic pain condition using a random-effects model. ${ }^{47}$ We will investigate the potential for statistical heterogeneity using visual inspection of the forest plots for each pairwise comparison as well as considering the magnitude of the between-study variance (tau-square) and the $\mathrm{I}^{2}$ statistic. $^{48}$

\section{Evaluation of transitivity}

Transitivity assumes that all interventions are 'jointly randomisable', and this assumption could be violated if interventions have different indications. ${ }^{49} 50$ In other words, transitivity may be violated if effect modifiers are not similarly distributed across the different comparisons in the network. We will examine the transitivity assumption using boxplots or percentages to visually inspect the distributions of the following potential effect modifiers: age, gender, chronic pain conditions, dose, duration and format methods. ${ }^{51}$ Networks of interventions will be formed and synthesised only when it will be reasonable to assume that any patient who meets our inclusion criteria is, in principle, equally likely to be randomised in any of the network nodes.

\section{Network meta-analysis}

NMA combines direct and indirect evidence for every comparison into a single effect size and therefore can increase the precision of the estimated relative effects. ${ }^{27} 285$ If there is no evidence for important intransitivity, we will then perform a random-effects NMA to simultaneously synthesise information on all available interventions for each outcome.

We will perform analyses using the multivariate NMA approach and produce presentation tools in Stata V.16 using network and network graphs packages. ${ }^{52}{ }^{53}$ We will also rank the competing interventions using the surface under the cumulative ranking curve (SUCRA) for the primary outcomes. A SUCRA of $100 \%$ implies that a treatment is certain to be the best and a SUCRA of $0 \%$ implies that it is certain to be the worst with respect to the studied outcome. ${ }^{53}$ We will present the available data using network diagrams in which the nodes will represent the treatments and the edges will represent the available direct comparisons. The size of nodes will be proportional to the number of participants allocated to each intervention and the thickness of the edges proportional to the number of studies comparing the respective interventions. We will also produce contribution matrices to investigate how much each study contributes to the estimation within a network.

In the case of an unconnected network (eg, two separate networks for pharmacological and non-pharmacological interventions or in the case of incomparable nodes of the examined interventions), we will conduct separate NMAs. We will also use a class-level model that allows estimating both class-level and intervention-level effects. ${ }^{54}$ This analysis will be conducted using r2jags, which accounts for variation in the definition of the nodes. ${ }^{55}$

\section{Assessment of coherence}

We will also evaluate statistically the assumption of coherence (ie, that direct and indirect evidence are in statistical agreement) using both a local and a global approach. Specifically, we will use the side-splitting method $^{56}$ that evaluates incoherence for every comparison with available direct evidence and the design-by-treatment interaction mode ${ }^{57}$ that tests incoherence in the entire network.

\section{Subgroup analyses}

Whenever feasible, independent NMAs for different groups of studies will be performed

- Within subgroups of chronic pain conditions (chronic neuropathic pain, chronic low back pain, chronic neck pain including whiplash-associated disorders, chronic widespread pain, fibromyalgia, myofascial pain syndromes, Ehlers-Danlos syndrome and hypermobility syndromes, and osteoarthritis).

- Within subgroups of gender and age defining two subgroups per variable: women versus men and younger ages $(\leq 49$ years old) versus older ages $(\geq 50$ years old).

- Within subgroups of measurements of RTW outcomes defining two subgroups: 'objective' (eg, records) versus self-reported measurements.

- Within subgroups of interventions defining two subgroups: combined interventions (two or more interventions in experimental arm) versus single interventions (monotherapies).

\section{Meta-regression analysis}

We plan to conduct meta-regression for clinically relevant subgroups or when important statistical heterogeneity or incoherence is detected. ${ }^{58}$ With this analysis, we will be able to examine the impact of important factors and effect modifiers on our results. We will use as covariates the following characteristics: age, gender, chronic pain conditions, dose, duration and format methods. We will assume (a) a common coefficient across all comparisons and (b) comparison-specific coefficients linked through the consistency assumption. All coefficients will be given non-informative priors. Each covariate will be included in a different meta-regression model. If enough studies are 
available, multivariable models will also be considered. To perform the meta-regressions, we will use r2jags. ${ }^{55}$

\section{Sensitivity analysis}

We plan to perform sensitivity analysis to assess the robustness of our results by excluding studies at high risk of bias and studies that have used imputations for missing outcome data.

\section{Small-study effects and publication bias}

The comparison-adjusted funnel plot $^{59}$ will be used to evaluate small-study effects at the network level. In case funnel plot asymmetry will be detected, we will use network meta-regression models to test its statistical significance. ${ }^{60}$ We will also assess the potential for publication bias, and if suspected, we will employ selection models. ${ }^{61}$

\section{Quality of evidence}

We will assess the overall quality of evidence for each treatment comparison (on the main outcomes) using the Confidence in Network Meta-Analysis (CINeMA) tool (https://cinema.ispm.unibe.ch) ${ }^{62}$ CINeMA allows for a quality of evidence judgement of a specific comparison based on the relative contributions of both direct and indirect evidence. ${ }^{62} 63$

\section{Patient and public involvement}

This is a meta-research project, and therefore there will be no patient's participation or public involvement in any of the study progress.

\section{Ethics and dissemination}

Ethical approval is not required for this type of study. The results of this study will be circulated as soon as they are available by a peer-review publication.

\section{DISCUSSION}

This systematic review and NMA will place RTW as a primary treatment goal for work-disabled individuals with CNMP, by addressing the efficacy of RTW interventions and supplying a summary of the evidence in terms of ranking of treatment effects and overall completeness and the applicability of the reported evidence. Hence, our study will make a substantial contribution to the evaluation of multiple RTW interventions in a severely affected group of patients and thereby will assist in optimising different chronic pain rehabilitation interventions. That means that this systematic review and NMA will indicate which of those available interventions, for example, medications, psychological, exercise therapies, multidisciplinary interventions, occupational therapy programmes, policy and healthcare system strategies/programmes, can decrease work absenteeism due to CNMP. Our findings could help guide future research evaluating the best treatment option for RTW, which in turn will be useful to improve pain rehabilitation and sustainable work conditions and to decrease work absence due to CNMP. Consequently, the results of this study would be applied in a fundamental clinical and research field providing inspiration for further research and setting prerequisites for future RCTs. Limitations of this study may be moderateto-large heterogeneity, risk of publication bias and important statistical incoherence, which in turn might limit the type of interventions that can be included in the analysis. Another limitation could also be the differences in social security systems in different countries and the lack of equal outcomes and data sources.

Contributors ED conceived the idea and designed the review. $E D, M B$ and $A C$ wrote the first draft of this protocol, and MB, BG, GL, FS, MS, TT, AC and ED were responsible for the revision of the draft. All authors contributed to developing the search strategy and registering the protocol. ED and AC were the guarantors. All authors scrutinised and approved the final manuscript. AC and ED are joint last authors.

Funding This research was supported by a grant to ED by Sinnescentrum, Region Östergötland, Sweden (No. SC-2019-00155-41). The funding body did not take part in the collection, analysis and interpretation of data; in the writing of the manuscript; or in the decision to submit the manuscript for publication.

Competing interests None declared.

Patient consent for publication Not required.

Provenance and peer review Not commissioned; externally peer reviewed.

Supplemental material This content has been supplied by the author(s). It has not been vetted by BMJ Publishing Group Limited (BMJ) and may not have been peer-reviewed. Any opinions or recommendations discussed are solely those of the author(s) and are not endorsed by BMJ. BMJ disclaims all liability and responsibility arising from any reliance placed on the content. Where the content includes any translated material, BMJ does not warrant the accuracy and reliability of the translations (including but not limited to local regulations, clinical guidelines, terminology, drug names and drug dosages), and is not responsible for any error and/or omissions arising from translation and adaptation or otherwise.

Open access This is an open access article distributed in accordance with the Creative Commons Attribution Non Commercial (CC BY-NC 4.0) license, which permits others to distribute, remix, adapt, build upon this work non-commercially, and license their derivative works on different terms, provided the original work is properly cited, appropriate credit is given, any changes made indicated, and the use is non-commercial. See: http://creativecommons.org/licenses/by-nc/4.0/.

ORCID iD

Elena Dragioti http://orcid.org/0000-0001-9019-4125

\section{REFERENCES}

1 Patel AS, Farquharson R, Carroll D, et al. The impact and burden of chronic pain in the workplace: a qualitative systematic review. Pain Pract 2012;12:578-89.

2 Goldberg DS, McGee SJ. Pain as a global public health priority. BMC Public Health 2011;11:770.

3 van Leeuwen MT, Blyth FM, March LM, et al. Chronic pain and reduced work effectiveness: the hidden cost to Australian employers. Eur J Pain 2006;10:161-6.

4 Bernfort L, Gerdle B, Rahmqvist M, et al. Severity of chronic pain in an elderly population in Sweden--impact on costs and quality of life. Pain 2015;156:521-7.

5 Phillips CJ, Harper C. The economics associated with persistent pain. Curr Opin Support Palliat Care 2011;5:127-30.

6 Stewart WF, Ricci JA, Chee E, et al. Lost productive time and cost due to common pain conditions in the US workforce. JAMA 2003;290:2443-54

7 Breivik H, Collett B, Ventafridda V, et al. Survey of chronic pain in Europe: prevalence, impact on daily life, and treatment. Eur J Pain 2006;10:287-333.

8 Mäntyselkä P, Kumpusalo E, Ahonen R, et al. Pain as a reason to visit the doctor: a study in Finnish primary health care. Pain 2001;89:175-80.

9 Nahin RL. Estimates of pain prevalence and severity in adults: United States, 2012. J Pain 2015;16:769-80. 
10 Hoy D, March L, Brooks P, et al. The global burden of low back pain: estimates from the global burden of disease 2010 study. Ann Rheum Dis 2014;73:968-74.

11 Hogan M-E, Taddio A, Katz J, et al. Incremental health care costs for chronic pain in Ontario, Canada: a population-based matched cohort study of adolescents and adults using administrative data. Pain 2016;157:1626-33.

12 Verbunt JA, Sieben J, Vlaeyen JWS, et al. A new episode of low back pain: who relies on bed rest? Eur J Pain 2008;12:508-16.

13 Franklin GM, Rahman EA, Turner JA, et al. Opioid use for chronic low back pain. Clin J Pain 2009;25:743-51.

14 Oliveira CB, Maher CG, Pinto RZ, et al. Clinical practice guidelines for the management of non-specific low back pain in primary care: an updated overview. Eur Spine J 2018;27:2791-803.

15 Sundstrup E, Seeberg KGV, Bengtsen E, et al. A systematic review of workplace interventions to rehabilitate musculoskeletal disorders among employees with physical Demanding work. J Occup Rehabil 2020:s10926-020-09879-x.

16 Cullen KL, Irvin E, Collie A, et al. Effectiveness of workplace interventions in return-to-work for musculoskeletal, pain-related and mental health conditions: an update of the evidence and messages for practitioners. J Occup Rehabil 2018;28:1-15.

17 Schaafsma F, Schonstein E, Whelan KM, et al. Physical conditioning programs for improving work outcomes in workers with back pain. Cochrane Database Syst Rev 2010;1:CD001822.

18 Norlund A, Ropponen A, Alexanderson K. Multidisciplinary interventions: review of studies of return to work after rehabilitation for low back pain. J Rehabil Med 2009;41:115-21.

19 Vogel N, Schandelmaier S, Zumbrunn T, et al. Return-To-Work coordination programmes for improving return to work in workers on sick leave. Cochrane Database Syst Rev 2017:3:CD011618.

20 Bültmann U, Franche R-L, Hogg-Johnson S, et al. Health status, work limitations, and return-to-work trajectories in injured workers with musculoskeletal disorders. Qual Life Res 2007;16:1167-78.

21 Marmot M, Allen J, Bell R, et al. Who European review of social determinants of health and the health divide. Lancet 2012;380:1011-29.

22 Rueda S, Chambers L, Wilson M, et al. Association of returning to work with better health in working-aged adults: a systematic review. Am J Public Health 2012;102:541-56.

23 Tavakoli-Fard N, Mortazavi S-A, Kuhpayehzadeh J, et al. Quality of life, work ability and other important indicators of women's occupational health. Int J Occup Med Environ Health 2016;29:77-84.

24 Marrone J, Swarbrick MA. Long-Term unemployment: a social determinant Underaddressed within community behavioral health programs. Psychiatr Serv 2020;71:appips201900522:745-8.

25 Wang $\mathrm{M}-\mathrm{C}$, Lin F-L, Su H-H, et al. Revisiting the relationship between suicide and unemployment in Mexico: evidence from linear and nonlinear Co-integration. Front Public Health 2020;8:60.

26 Kamper SJ, Apeldoorn AT, Chiarotto A, et al. Multidisciplinary biopsychosocial rehabilitation for chronic low back pain: cochrane systematic review and meta-analysis. BMJ 2015;350:h444.

27 Cipriani A, Furukawa TA, Salanti G, et al. Comparative efficacy and acceptability of 12 new-generation antidepressants: a multipletreatments meta-analysis. Lancet 2009;373:746-58.

28 Leucht S, Chaimani A, Cipriani AS, et al. Network meta-analyses should be the highest level of evidence in treatment guidelines. Eur Arch Psychiatry Clin Neurosci 2016;266:477-80.

29 Koechlin $\mathrm{H}$, Whalley B, Welton NJ, et al. The best treatment option(s) for adult and elderly patients with chronic primary musculoskeletal pain: a protocol for a systematic review and network meta-analysis. Syst Rev 2019;8:269.

30 Rouse B, Chaimani A, Li T. Network meta-analysis: an introduction for clinicians. Intern Emerg Med 2017;12:103-11.

31 Moher D, Shamseer L, Clarke M, et al. Preferred reporting items for systematic review and meta-analysis protocols (PRISMA-P) 2015 statement. Syst Rev 2015;4:1.

32 Chaimani A, Caldwell DM, Li T, et al. Additional considerations are required when preparing a protocol for a systematic review with multiple interventions. J Clin Epidemiol 2017;83:65-74.

33 Hutton B, Salanti G, Caldwell DM, et al. The PRISMA extension statement for reporting of systematic reviews incorporating network meta-analyses of health care interventions: checklist and explanations. Ann Intern Med 2015;162:777-84.

34 Williams AC, Craig KD. Updating the definition of pain. Pain 2016;157:2420-3.

35 Dragioti E, Dong H-J, Larsson B, et al. Reported outcomes in published systematic reviews of interdisciplinary pain treatment: protocol for a systematic overview. JMIR Res Protoc 2020;9:e17795.
36 de Boer AG, Taskila TK, Tamminga SJ, et al. Interventions to enhance return-to-work for cancer patients. Cochrane Database Syst Rev 2015;9:CD007569.

37 Uhl RL, Roberts TT, Papaliodis DN, et al. Management of chronic musculoskeletal pain. J Am Acad Orthop Surg 2014;22:101-10.

38 Donker-Cools BHPM, Daams JG, Wind H, et al. Effective return-towork interventions after acquired brain injury: a systematic review. Brain Inj 2016;30:113-31.

39 van Vilsteren $\mathrm{M}$, van Oostrom $\mathrm{SH}$, de Vet HCW, et al. Workplace interventions to prevent work disability in workers on sick leave. Cochrane Database Syst Rev 2015;10:CD006955.

40 Nolan SJ, Hambleton I, Dwan K. The use and reporting of the cross-over study design in clinical trials and systematic reviews: a systematic assessment. PLoS One 2016;11:e0159014.

41 Higgins JPT, Eldridge S, Li T. Including variants on randomized trials. In: Cochrane Handbook for systematic reviews of interventions, 2019: 569-93.

42 Furukawa TA, Salanti G, Atkinson LZ, et al. Comparative efficacy and acceptability of first-generation and second-generation antidepressants in the acute treatment of major depression: protocol for a network meta-analysis. BMJ Open 2016;6:e010919.

43 Higgins JPT, Deeks JJ. Chapter 7: selecting studies and collecting data. In: Green S, ed. Cochrane Handbook for systematic reviews of interventions version 5.1.0 (updated March 2011. The Cochrane Collaboration, 2011.

44 Wan X, Wang W, Liu J, et al. Estimating the sample mean and standard deviation from the sample size, median, range and/or interquartile range. BMC Med Res Methodol 2014;14:135.

45 Sterne JAC, Savović J, Page MJ, et al. Rob 2: a revised tool for assessing risk of bias in randomised trials. BMJ 2019;366:14898.

46 Chaimani A, Caldwell DM, Li T, et al. Cochrane Handbook for systematic reviews of interventions version 6.0 (updated July 2019). Cochrane Database Syst Rev 2019.

47 DerSimonian R, Laird N. Meta-Analysis in clinical trials. Control Clin Trials 1986;7:177-88.

48 Higgins JPT, Thompson SG, Deeks JJ, et al. Measuring inconsistency in meta-analyses. BMJ 2003;327:557-60.

49 Efthimiou O, Debray TPA, van Valkenhoef G, et al. GetReal in network meta-analysis: a review of the methodology. Res Synth Methods 2016;7:236-63.

50 Cipriani A, Higgins JPT, Geddes JR, et al. Conceptual and technical challenges in network meta-analysis. Ann Intern Med 2013;159:130-7.

51 Salanti G. Indirect and mixed-treatment comparison, network, or multiple-treatments meta-analysis: many names, many benefits, many concerns for the next generation evidence synthesis tool. Res Synth Methods 2012;3:80-97.

52 White IR. Network meta-analysis. Stata J 2015;15:951-85.

53 Chaimani A, Salanti G. Visualizing assumptions and results in network meta-analysis: the network graphs package. Stata $J$ 2015;15:905-50.

54 Del Giovane C, Vacchi L, Mavridis D, et al. Network meta-analysis models to account for variability in treatment definitions: application to dose effects. Stat Med 2013;32:25-39.

55 Package 'R2jags'. Using R to Run 'JAGS', 2020. Available: https:// cran.r-project.org/web/packages/R2jags/R2jags.pdf

56 Dias S, Welton NJ, Caldwell DM, et al. Checking consistency in mixed treatment comparison meta-analysis. Stat Med 2010;29:932-44.

57 Higgins JPT, Jackson D, Barrett JK, et al. Consistency and inconsistency in network meta-analysis: concepts and models for multi-arm studies. Res Synth Methods 2012;3:98-110.

58 Higgins JPT, Thomas J, Chandler J, et al. Cochrane Handbook for systematic reviews of interventions, 2019

59 Chaimani A, Higgins JPT, Mavridis D, et al. Graphical tools fo network meta-analysis in STATA. PLoS One 2013;8:e76654.

60 Chaimani A, Salanti G. Using network meta-analysis to evaluate the existence of small-study effects in a network of interventions. Res Synth Methods 2012;3:161-76.

61 Mavridis D, Welton NJ, Sutton A, et al. A selection model for accounting for publication bias in a full network meta-analysis. Stat Med 2014;33:5399-412.

62 Nikolakopoulou A, Higgins JPT, Papakonstantinou T, et al. Cinema: an approach for assessing confidence in the results of a network meta-analysis. PLoS Med 2020;17:e1003082.

63 Salanti G, Del Giovane C, Chaimani A, et al. Evaluating the quality of evidence from a network meta-analysis. PLoS One 2014;9:e99682. 